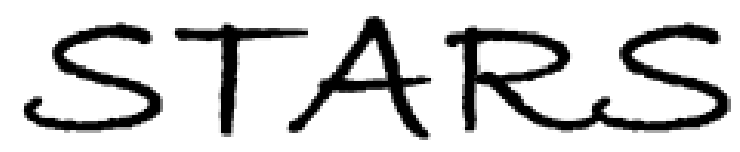

University of Central Florida

STARS

$1-1-2006$

\title{
Time-dependent quantum transport and nonquasistatic effects in carbon nanotube transistors
}

Yupeng Chen

University of Central Florida

Yigian Ouyang

Jing Guo

Thomas X. Wu

University of Central Florida

Find similar works at: https://stars.library.ucf.edu/facultybib2000

University of Central Florida Libraries http://library.ucf.edu

This Article is brought to you for free and open access by the Faculty Bibliography at STARS. It has been accepted for inclusion in Faculty Bibliography 2000 s by an authorized administrator of STARS. For more information, please contact STARS@ucf.edu.

\section{Recommended Citation}

Chen, Yupeng; Ouyang, Yigian; Guo, Jing; and Wu, Thomas X., "Time-dependent quantum transport and nonquasistatic effects in carbon nanotube transistors" (2006). Faculty Bibliography 2000s. 4671.

https://stars.library.ucf.edu/facultybib2000/4671

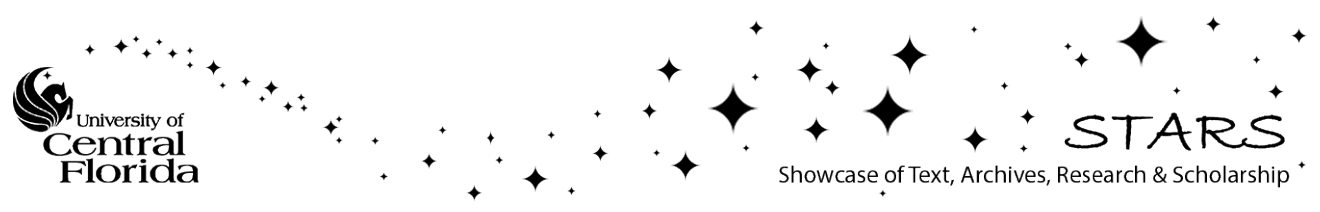




\section{Time-dependent quantum transport and nonquasistatic effects in carbon nanotube transistors}

Cite as: Appl. Phys. Lett. 89, 203122 (2006); https://doi.org/10.1063/1.2388881

Submitted: 03 August 2006. Accepted: 09 October 2006. Published Online: 17 November 2006

Yupeng Chen, Yijian Ouyang, Jing Guo, and Thomas X. Wu

\section{ARTICLES YOU MAY BE INTERESTED IN}

Poole-Frenkel-effect as dominating current mechanism in thin oxide films-An illusion?!

Journal of Applied Physics 117, 215103 (2015); https://doi.org/10.1063/1.4921949

\section{Applied Physics Letters}

Mid-IR and THz frequency combs special collection

\section{Read Now!}




\title{
Time-dependent quantum transport and nonquasistatic effects in carbon nanotube transistors
}

\author{
Yupeng Chen \\ School of Electrical Engineering and Computer Science, University of Central Florida, Orlando, \\ Florida 32816 \\ Yijian Ouyang and Jing Guo \\ Department of Electrical and Computer Engineering, University of Florida, Gainesville, Florida 32611 \\ Thomas X. Wu ${ }^{\mathrm{a})}$ \\ School of Electrical Engineering and Computer Science, University of Central Florida, Orlando, \\ Florida 32816
}

(Received 3 August 2006; accepted 9 October 2006; published online 17 November 2006)

\begin{abstract}
Nonquasistatic effects in ac characteristics of carbon nanotube field-effect transistors are examined by solving a full time-dependent, open-boundary Schrödinger equation. The nonquasistatic characteristics, such as the finite channel charging time, and the dependence of small signal transconductance and gate capacitance on the frequency, are explored. The validity of the widely used quasistatic approximation is examined. The results show that the quasistatic approximation overestimates the transconductance and gate capacitance at high frequencies, but gives a more accurate value for the intrinsic cutoff frequency over a wide range of bias conditions. (C) 2006 American Institute of Physics. [DOI: 10.1063/1.2388881]
\end{abstract}

Carbon nanotubes (CNTs) have been extensively explored for potential nanoelectronics applications due to its excellent electrical properties. ${ }^{1,2}$ Recently, ac characteristics of carbon nanotube field-effect transistors (FETs) are attracting extensive research interests due to their high mobility and near ballistic transport. ${ }^{3}$ Appenzeller and Frank performed the pioneering experimental work on the ac characteristics of CNTFETs with a frequency up to $580 \mathrm{MHz} .{ }^{4}$ The measurements with a frequency of $2.6 \mathrm{GHz}$ (Ref. 5) and $10 \mathrm{GHz}$ (Ref. 6) were subsequently reported. Very recently, a five stage CNT ring oscillators with a frequency of $52 \mathrm{MHz}$ has been deomonstrated ${ }^{7}$ and ac characterization of a CNTFET up to $50 \mathrm{GHz}$ was reported. ${ }^{8}$ The ultimate goal is to benefit from the intrinsic high speed of CNTFET and achieve the theoretically predicted terahertz intrinsic frequency. Selfconsistent, quasistatic (QS) quantum simulations have been applied to assess the high-frequency performance. ${ }^{9,10}$ The validity of the QS approximation at very high frequencies, however, should be questioned. Previous studies have shown that nonquasistatic (NQS) effects play an important role on the characteristics of conventional high-speed transistors. ${ }^{11}$ Little, however, is known about the NQS effects in carbon nanotube transistors.

In this letter, NQS effects in ballistic CNTFETs are investigated by solving a full time-dependent open boundary Schrödinger equation for CNTFETs using the finite difference time domain (FDTD) method. ${ }^{12}$ The dependence of small signal transconductance and gate capacitance on the frequency of the applied bias is examined. The intrinsic cutoff frequency $\left(f_{T}\right)$, a device metric important for radiofrequency (rf) applications, is computed using the full timedependent simulations. The validity of the widely used QS approximation is examined.

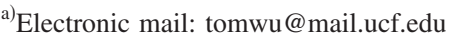

A coaxially gated CNTFET is modeled. The coaxially gate geometry is ideal for suppressing short channel effects and for simplifying the treatment of self-consistent electrostatics. ${ }^{12}$ The qualitative conclusions of this work also apply to planar gated CNTFETs. The intrinsic $(17,0)$ zigzag CNT channel has a channel length of $L_{\mathrm{ch}}=20 \mathrm{~nm}$ and a diameter of $d_{\mathrm{CNT}} \approx 1.33 \mathrm{~nm}$, which results in a band gap of $E_{g} \approx 0.65 \mathrm{eV}$. The Schottky barrier height $\Phi_{B}$ between the metal source and drain contacts and the intrinsic CNT channel depends on the contact material and tube diameter. In this work, we use $\Phi_{B}=0.33 \mathrm{eV}$. Because the conduction and valence bands of CNTs are symmetric, $n$-type conduction is modeled for simplicity. The high- $\kappa$ gate insulator thickness is $t_{\mathrm{ms}}=2.5 \mathrm{~nm}$ and the dielectric constant $\kappa \approx 25$. The relative effective mass of the CNT is $0.06{ }^{13}$ The simulations are performed at the room temperature, $T=300 \mathrm{~K}$. A power supply voltage of $V_{\mathrm{DD}}=0.5 \mathrm{~V}$. An ac signal is applied at the gate to modulate the transport of the carriers.

In order to treat time-dependent transport in the channel and quantum tunneling through the metal/CNT Schottky barriers, we solve the time-dependent Schrödinger equation by extending the FDTD method as described in Ref. 14 with the treatment of the open boundary condition. ${ }^{15}$ When the frequency is much smaller than the speed of the wave propagation $\left(\sqrt{5}\right.$ times Fermi velocity $\left.{ }^{16}\right)$ divided by the channel length (which is $\sim 100 \mathrm{THz}$ for the simulated device with a channel length of $20 \mathrm{~nm}$ ) electrostatic simulation can be used instead of full-wave electromagnetics simulation. Because the frequency range of interest is much smaller than $\sim 100 \mathrm{THz}$, the time-dependent quantum transport equation is solved self-consistently with the Poisson equation. The effects of kinetic inductance are included because of the full time-dependent simulation. ${ }^{17}$ Ballistic transport in the CNT channel is assumed. An effective mass description of the CNT band structure is used. ${ }^{18}$ The parasitic capacitances between the gate electrode and source (drain) contact, which significantly decrease the operation frequency of the devices 

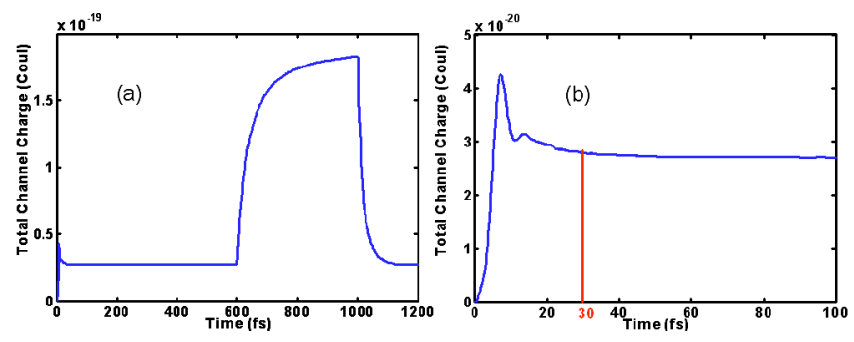

FIG. 1. (a) Switching transient characteristics of the channel charge with the applied rectangular pulse ( $V_{\mathrm{GS}}$ from 0 to 0.5 to $0 \mathrm{~V}$, between 600 and $1000 \mathrm{fs}$ ) to the gate. (b) Power-on transient characteristics of the channel charge. The transistor is turned on $\left(V_{\mathrm{DS}}\right.$ from 0 to $\left.0.5 \mathrm{~V}\right)$ at $t=0$. The settling time is $30 \mathrm{fs}$.

in the current experiments, are neglected. The simulation results based on the above assumptions set the high-frequency performance limit of CNTFETs. (The effects of parasitic capacitances and scattering are briefly discussed later.)

The time-dependent drain to source current and charge density are calculated by the probability current density and probability density ${ }^{19}$ from the solved time-dependent wave function $\psi(t)$ as Eqs. (1a) and (1b), where $d E / d k$ is calculated from $E-k$ relation in contacts, $\hbar$ is the reduced Planck constant, and $m^{*}$ is the effective mass.

$$
\begin{aligned}
& i_{\mathrm{DS}}(t)=\frac{2 e \hbar}{\pi m^{*}} \int \frac{1}{(d E / d k)} \operatorname{Im}\left(\psi(t)^{*} \nabla \psi(t)\right) d E, \\
& \rho_{v}(t)=\frac{2 e}{\pi} \int \frac{1}{(d E / d k)}|\psi(t)|^{2} d E .
\end{aligned}
$$

In quasistatic approximation, it is assumed that the charge in a transistor channel responds instantaneously as the terminal voltage is applied. We explore the finite time that takes to charge and discharge the CNT channel using the time-dependent simulation. Figure 1 plots the simulated total amount of charge in the channel as a function of time when a rectangular pulse signal is applied on the gate, where Fig. 1(a) plots the time period with the gate voltage pulse and Fig. 1(b) plots the power-on (drain voltage step) time period. At $t=0$, the device is powered on with $V_{\mathrm{DS}}=0.5 \mathrm{~V}$ and $V_{\mathrm{GS}}$ $=0 \mathrm{~V}$. The channel charge goes to steady state after an overshoot time period, which is caused by zero rising time of the drain voltage step. The approximate rising time (for the charge to increase to the steady state value) is about $30 \mathrm{fs}$. The finite charging time is due to the facts that the charge in the channel is supplied by carrier injection from the source and that the source-injected carriers travel at a speed below the band-structure-limited speed. In order to characterize how fast carriers travel to charge the channel, an effective velocity, which is defined as the channel length divided by the rising time, is computed to be $\sim 6.67 \times 10^{5} \mathrm{~m} / \mathrm{s}$. It is in the same order of magnitude but still smaller than the Fermi velocity in metallic CNTs (about $9.6 \times 10^{5} \mathrm{~m} / \mathrm{s}$ ) because of the following two reasons. First, tunneling through the Schottky barrier at the source end of the channel slows down the carriers. Second, carriers populate the bottom portion of a semiconducting $E-k$ relation, which has a smaller bandstructure-limited velocity.

We next explore the NQS effect in the small signal configurations. A small signal sinusoidal wave with amplitude of $10 \mathrm{mV}$ is superimposed with dc biasing on the gate. Figure 2(a) shows the resulting time-dependent small signal drain to

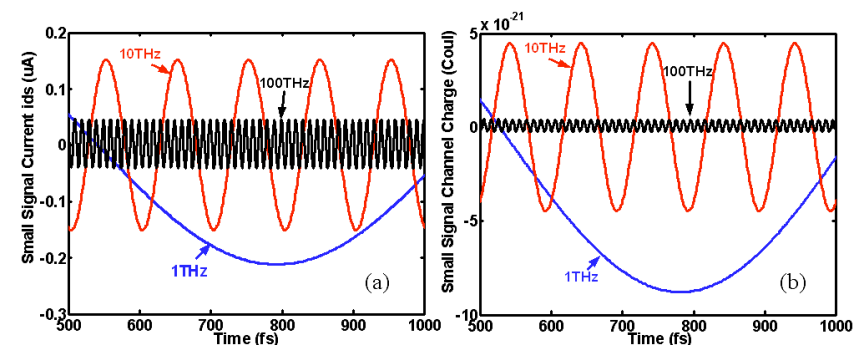

FIG. 2. (a) Small signal time dependent drain to source current. (b) Small signal time dependent channel charge at $V_{\mathrm{DS}}=0.5 \mathrm{~V}, V_{\mathrm{GS}}=0.5 \mathrm{~V}$, and $V_{\mathrm{GS}}$ $=10 \mathrm{mV}$.

source current for different frequencies $(1,10$, and $100 \mathrm{THz})$. With the increasing of the frequency, the small signal amplitude of the ac current is gradually decreased. This is because the transit time is comparable to the period of the small signal at high frequency. At very high frequency, the small signal voltage on the gate cannot modulate the transport current, which means that the gate will lose the control of the device at such high frequency. Similar behavior can be expected for time dependent channel charge as shown in Fig. 2(b). At very high frequency, the potential in the channel will change before the channel charge accumulates to the new level. So, the channel charge is almost constant, which is close to dc charge.

Based on the results of Fig. 2, we can obtain the frequency dependent small signal transconductance and gate capacitance as shown in Fig. 3. Comparing these NQS values with QS simulation results, ${ }^{9,10}$ we find that up to the frequency of $\sim 1 \mathrm{THz}$, the results of NQS approach are close to that of QS approach. However, when the frequency is above $1 \mathrm{THz}$, the NQS results show that the small signal transconductance and gate capacitance decrease accordingly, while the QS approach cannot show frequency dependent results. As indicated in Fig. 3(a), 3 dB bandwidth of the transconductance is around $10 \mathrm{THz}$, which is a very important specification for the amplifier applications.

An important performance metric for high frequency analog application is the cutoff frequency. Next we examine the validity of QS approximation for computing the cutoff frequency. Figure 4(a) shows how the cutoff frequency is computed in a time-dependent simulation. It plots the gate current and the source-drain current as a function of the frequency of the applied gate voltage. As frequency increases, the impedance of the gate capacitor decreases and the gate current increases. On the other hand, when the frequency increases, the source-drain current decreases because the transconductance decreases as a function of the frequency as shown in Fig. 3. The cutoff frequency, which is defined as the unit current gain frequency, is $4.16 \mathrm{THz}$ as shown in Fig.
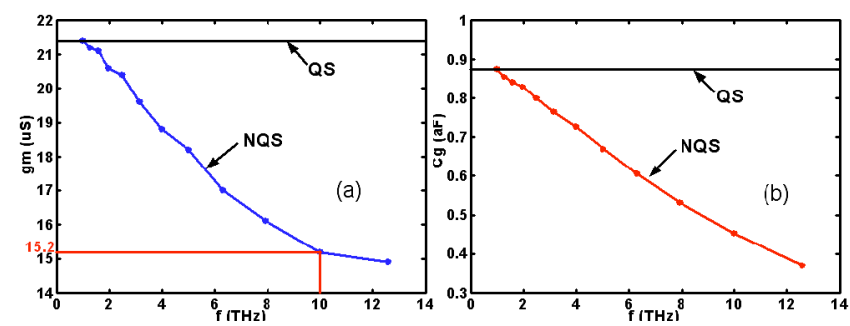

FIG. 3. (a) ac transconductance and (b) gate capacitance vs frequency at $V_{\mathrm{DS}}=0.5 \mathrm{~V}$ and $V_{\mathrm{GS}}=0.5 \mathrm{~V}$. 

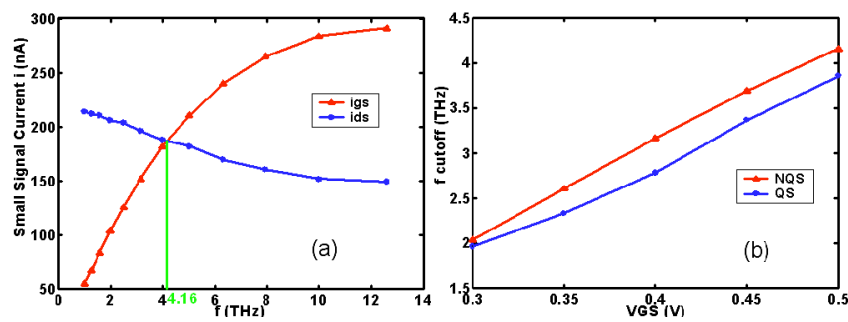

FIG. 4. (a) Small signal drain to source and gate to source current vs frequency at $V_{\mathrm{DS}}=0.5 \mathrm{~V}$ and $V_{\mathrm{GS}}=0.5 \mathrm{~V}$. (b) Cutoff frequency vs gate voltage at $V_{\mathrm{DS}}=0.5 \mathrm{~V}$ by NQS (line with triangle) and QS (circled line) approaches.

4(a). This value is well below $100 \mathrm{THz}$, which justified the approximation that Poisson equation applies.) Using the QS approach, ${ }^{9,10}$ the intrinsic cutoff frequency can be computed as $g_{m} /\left(2 \pi C_{g}\right)$, where $g_{m}$ and $C_{g}$ are the dc transconductance and gate capacitance, respectively. The intrinsic cutoff frequency $f_{T}$ computed by the QS approximation is $3.86 \mathrm{THz}$, which is different from that computed by time-dependent simulation by about $7 \%$. We notice that although the QS approximation overestimates the transconductance at $f_{T}$ by $14 \%$ and the intrinsic gate capacitance by $21 \%$, it results in an intrinsic cutoff frequency much closer to that computed by the time-dependent simulation due to the following reason. As frequency increases, the simultaneous decrease of the transconductance and the gate capacitance makes their ratio (which determines $f_{T}$ ) less affected. The QS estimation of the cutoff frequency is expected to be even more accurate when a state-of-the-art experimental CNTFET is considered, which operates well below its high-frequency performance limit because the parasitic capacitances between the gate electrode and source (drain) electrode dominate. ${ }^{10}$ Because the parasitic capacitances are nearly bias independent, the frequency dependent gate capacitance will have little effect on the total capacitance. Since the extrinsic cutoff frequency is orders of magnitude smaller than the intrinsic cutoff frequency (following Refs. 20 and 21), we simulate a Schottky-barrier FET with metal nanowire source/drain contacts and a very thin gate electrode. The parasitic capacitance is about four times larger than the intrinsic capacitance and the cutoff frequency is $\sim 20 \%$ of the intrinsic value because the simulated channel length is very short $(20 \mathrm{~nm})$. As the channel length increases, the intrinsic component becomes more dominant. For example, the parasitic capacitance is less than $10 \%$ of the intrinsic gate capacitance and the cutoff frequency is $\sim 90 \%$ of the intrinsic value when the channel length is $1 \mu \mathrm{m}$. The transconductance and the gate capacitance are close to the QS estimations at such a low frequency. In this case, the QS approach gives a good estimation of the extrinsic cutoff frequency.

The above comparison is performed at a specific bias condition. Next, we explore the voltage dependence. Figure 4(b) plots $f_{T}$ computed by QS approximation and that computed from the time-dependent simulation as a function of the applied dc gate voltage. The qualitative feature of the curve by QS simulation, ${ }^{9,10}$ is similar to that of timedependent simulation. The intrinsic cutoff frequency of the CNTFET increases with gate biasing when the gate voltage is relatively small and tends to remain constant as $V_{G}$ further increases but still smaller than $V_{D}$. Over the whole simulated bias regime, the difference between the quasistatic approximation and the time-dependent simulation is less than $7 \%$, which indicates the validity of quasistatic approximation for computing the cutoff frequency over a wide range of bias conditions.

Ballistic transport is assumed in this study. At low $V_{D}$, acoustic phonon scattering (with a mean free path of $\sim 1 \mu \mathrm{m}$ ) dominates and the simulated $20 \mathrm{~nm}$ long channel is near ballistic. At high $V_{D}$, optical and zone boundary phonon scattering (with a mean free path of $\sim 10 \mathrm{~nm}$ ) dominates, which occur even in a $20 \mathrm{~nm}$ long channel. The NQS effects are due to the fact that carriers travel at a finite average velocity, so that the device cannot respond instantaneously. We, therefore, expect that NQS effects become important at a lower frequency in the presence of scattering because the average carrier velocity decreases. The effect of scattering warrants a careful future study, but this work indicates that at the ballistic limit, the NQS effect becomes important when the frequency approaches the intrinsic cutoff frequency.

In summary, a full time-dependent, open-boundary Schrödinger equation is self-consistently solved for a CNTFET at the ballistic limit using FDTD method. NQS effects are explored and the validity of the widely used QS approximation is examined. The results show that the intrinsic gate capacitance and transconductance significantly decreases as the frequency increases to a value comparable to the intrinsic cutoff frequency. The channel charging time is limited by the band-structure-limited velocity. The quasistatic approximation gives a much more accurate value for the intrinsic cutoff frequency over a wide range of bias conditions than for the gate capacitance and transconductance.

${ }^{1}$ P. Avouris, J. Appenzeller, R. Martel, and S. J. Wind, Proc. IEEE 91, 1772 (2003).

${ }^{2}$ P. L. McEuen, M. S. Fuhrer, and H. K. Park, IEEE Trans. Nanotechnol. 1, 78 (2002).

${ }^{3}$ P. J. Burke, Solid-State Electron. 48, 1981 (2004).

${ }^{4}$ J. Appenzeller and D. J. Frank, Appl. Phys. Lett. 84, 1771 (2004).

${ }^{5}$ S. D. Li, Z. Yu, S. F. Yen, W. C. Tang, and P. J. Burke, Nano Lett. 4, 753 (2004).

${ }^{6}$ X. Huo, M. Zhang, P. C. H. Chan, Q. Chang, and Z. K. Tang, Tech. Dig. - Int. Electron Devices Meet. 2004, 691.

${ }^{7}$ Z. Chen, J. Appenzeller, Y. Lin, J. Sippel-Oakley, A. G. Rinzler, J. Tang, S. J. Wind, P. M. Solomon, and P. Avouris, Science 311, 1735 (2006).

${ }^{8}$ S. Rosenblatt, H. Lin, V. Sazonova, S. Tiwari, and P. L. McEuenb, Appl. Phys. Lett. 87, 153111 (2005).

${ }^{9}$ L. Castro, D. John, D. L. Pulfrey, M. Pourfath, A. Gehring, and H. Kosina, IEEE Trans. Nanotechnol. 4, 699 (2005).

${ }^{10}$ J. Guo, S. Hasan, A. Javey, G. Bosman, and M. Lundstrom, IEEE Trans. Nanotechnol. 4, 715 (2005).

${ }^{11}$ M. Chan, K. Y. Hui, and C. Hu, IEEE Trans. Electron Devices 45, 834 (1998).

${ }^{12}$ D. L. John, L. C. Castro, J. P. Clifford, and D. L. Pulfrey, IEEE Trans. Nanotechnol. 2, 175 (2003).

${ }^{13}$ G. L. Zhao, D. Bagayoko, and L. Yang, Phys. Rev. B 69, 245416 (2004).

${ }^{14}$ A. Soriano, E. A. Navarro, J. A. Porti, and V. Such, J. Appl. Phys. 95, 8011 (2004).

${ }^{15}$ T. Fevens and H. Jiang, SIAM J. Sci. Comput. (USA) 21, 255 (1999).

${ }^{16}$ A. Naeemi, R. Sarvari, and J. D. Meindl, IEEE Electron Device Lett. 26, 84 (2005).

${ }^{17}$ S. Salahuddin, M. Lundstrom, and S. Datta, IEEE Trans. Electron Devices 52, 1734 (2005).

${ }^{18}$ D. L. John, L. C. Castro, P. J. S. Pereira and D. L. Pulfrey, Proceedings of the NSTI Nanotech., edited by M. Laudon and B. Romanowicz (NSTI, Boston, 2004), Vol. 3, pp. 65-68.

${ }^{19}$ S. Datta, Quantum Transport Atom to Transistor, 2nd ed. (Cambridge University Press, New York, 2005).

${ }^{20}$ Y. Wu, J. Xiang, C. Yang, W. Lu, and C. M. Lieber, Nature (London) 430, 61 (2004).

${ }^{21}$ K. Alam and R. K. Lake, J. Appl. Phys. 100, 024317 (2006). 